\title{
Development of an Ewing sarcoma cell line with resistance to EWS-FLI1 inhibitor YK-4-279
}

\author{
ERIN CONN, SARAH HOUR, DAVID ALLEGAKOEN, GARRETT GRAHAM, JEFF PETRO, \\ MARILYN KOUASSI-BROU, SUNG HYEOK HONG, SARAVANA SELVANATHAN, \\ HAYDAR ÇELIK, JEFFREY TORETSKY and AYKUT ÜREN
}

Department of Oncology, Georgetown University Medical Center, Georgetown University, Washington, DC 20057, USA

Received July 17, 2019; Accepted October 28, 2019

DOI: $10.3892 / \mathrm{mmr} .2020 .10948$

\begin{abstract}
Despite Ewing sarcoma (ES) being the second most common pediatric malignancy of bone and soft tissue, few novel therapeutic approaches have been introduced over the past few decades. ES contains a pathognomonic chromosomal translocation that leads to a fusion protein between EWSRI and an ets family member, most often FLI1. EWS-FLI1 is the most common type of fusion protein and is a well-vetted therapeutic target. A small molecule inhibitor of EWS-FLI1, YK-4-279 (YK) was developed with the intention to serve as a targeted therapy option for patients with ES. The present study investigated resistance mechanisms by developing an ES cell line specifically resistant to YK. The ES cell line A4573 was treated with YK to create resistant cells by long term continuous exposure. The results revealed that resistance in A4573 was robust and sustainable, with a $>27$-fold increase in $\mathrm{IC}_{50}$ lasting up to 16 weeks in the absence of the compound. Resistant ES cells were still sensitive to standard of care drugs, including doxorubicin, vincristine and etoposide, which may be valuable in future combination treatments in the clinic. Resistant ES cells revealed an increased expression of CD99. RNA sequencing and qPCR validation of resistant ES cells confirmed an increased expression of ANO1, BRSK2 and IGSF21, and a reduced expression of COL24A1, PRSS23 and RAB38 genes. A functional association between these genes and mechanism of resistance remains to be investigated. The present study created a cell line to investigate YK resistance.
\end{abstract}

\section{Introduction}

Ewing sarcoma (ES) is a malignancy of the bones and soft tissue that affects children and adolescents. Approximately 300 new

Correspondence to: Dr Aykut Üren, Department of Oncology, Georgetown University Medical Center, Georgetown University, 3970 Reservoir Road, NW, Washington, DC 20057, USA

E-mail: au26@georgetown.edu

Key words: Ewing sarcoma, YK-4-279, resistance, EWS-FLI1, CD99, ANO1 cases are diagnosed each year in the USA, and it is the second most common bone and soft tissue cancer behind osteosarcoma. Current standard of care for ES patients includes control of all sites of disease with surgery and/or radiation for localized disease, usually combined with a multidrug chemotherapy regimen consisting of doxorubicin, vincristine, cyclophosphamide, etoposide and ifosfamide. Approximately two thirds of patients present with localized disease at the time of diagnosis, and 5-year survival for these patients is $>70 \%$ (1). For the one third of patients that present with metastatic disease at the time of diagnosis or patients with relapse, 5-year survival rates are below $30 \%$ (1).

Approximately $85 \%$ of all ES cases contain a balanced chromosomal translocation [t(11;22)(q24;q12)] between the ES breakpoint region 1 (EWSRl) gene and the Friend leukemia integration site 1 (FLII) gene (2). This fusion gene produces EWS-FLI1, a true chimeric protein, with the amino-terminal portion being derived from EWS and the carboxy-terminal portion being derived from FLI1 $(3,4)$. EWS is a member of the FET family of DNA and RNA binding proteins, and FLI1 is a member of the E26 transformation specific (ETS) family of transcription factor. Expression of EWS-FLI1 results in transformation (5-7) and is a critical driver of ES malignancy as well as ES cell survival $(3,8,9)$.

EWS-FLI1 exhibits multiple functions in ES that contribute to oncogenesis, including transcription and post-transcriptional mRNA splicing. The DNA binding domain of FLI1 is present in the fusion and binds to DNA at ETS binding sites containing GGAA motifs, while the EWS portion of the fusion protein functions as a transcriptional activator $(8,10)$. Additionally, EWS-FLI1 also binds to RNA helicase A (RHA/DHX9), a transcriptional coactivator, and localizes to promoter regions containing the ETS binding GGAA motifs where it then acts as a transcription factor and regulates gene expression (11). The transcriptional profile induced by EWS-FLI1 promotes oncogenic cellular processes. EWS-FLI1 has also been implicated in RNA processing events. EWS-FLI1 is capable of directly binding to RNA and inhibiting the helicase function of RHA, potentially altering RNA metabolism (12).

One of the first reports of EWS-FLI1 being actively involved in splicing mechanisms demonstrated that EWS-FLI1 inhibited splicing from occurring by interfering with the activity of serine-arginine splicing factors, thereby yielding 
alternatively spliced transcripts (13). EWS-FLI1 interacts with several spliceosome proteins including U1, U2, U5, and U4/6, which play a role in $5^{\prime}$ splice site selection (14-16). EWS-FLI1 expression induces expression changes as well as isoform shifts of several genes where it also differentially regulates gene expression including cyclin D1 and TERT $(16,17)$. The alternatively spliced isoforms of these genes produced by EWS-FLI1 expression display enhanced functional capabilities that increase the oncogenic potential of the cell.

YK-4-279 (YK) is a small molecule that was synthesized from a lead compound discovered by a surface plasmon resonance screen for direct binding to EWS-FLI1 $(18,19)$. YK disrupts the interaction between EWS-FLI1 and RHA, inhibiting transcription of target genes and restoring RHA helicase activity $(12,18)$. YK also blocks the interaction between EWS-FLI1 and members of the spliceosome, indicating that treatment with the compound also effectively targets the splicing modulation of EWS-FLI1. Importantly, YK effectively inhibits xenograft tumor growth in vivo (20). A synergistic relationship exists between YK and the standard of care chemotherapeutic agent vincristine in vitro, providing further evidence that this drug has high clinical value in the context of ES (21). YK is a chiral molecule, with the (S) enantiomer $[(\mathrm{S})-\mathrm{YK}]$ as the active enantiomer. $\mathrm{YK}$ is also effective at inhibiting other members of the ETS family of transcription factors such as ERG and ETV1 in prostate cancer (22-24), and may be of clinical value in other diseases such as neuroblastoma $(25,26)$.

An analog of YK called TK216 is currently undergoing a phase 1 clinical trial, and it appears likely that it will move forward to phase 2 (27). With any targeted agent, there is a possibility that resistance to the drug may develop in patients. We developed an ES cell line which acquired resistance to the (S) enantiomer of YK (referred to herein as YK) with the goals of better understanding the biology of ES cells after prolonged exposure to YK and identifying any potentially targetable changes in cells which have acquired resistance. In the future, tumor samples can be collected from patients with relapse and compared to cell line data from this manuscript. If a similar gene expression pattern is observed, the YK resistant cell line generated here may become a useful tool to study resistance mechanisms in this disease.

\section{Materials and methods}

Compounds. All compounds were dissolved in 100\% DMSO (ThermoFisher Scientific, Inc.). YK was obtained from AMRI Global. Etoposide, imatinib, and vincristine were purchased from Selleckchem. Doxorubicin and verapamil were purchased from MilliporeSigma.

Cell culture and development of $Y K$ resistant cell lines. A4573 sensitive and resistant (A4573-R) cell lines were maintained in RPMI (Thermo Fisher Scientific, Inc.) with 10\% FBS (MilliporeSigma) and $10 \mathrm{mM}$ HEPES (Thermo Fisher Scientific, Inc.). TC71 sensitive and resistant (TC71-R) cell lines were maintained in RPMI with $10 \%$ FBS. TC71-R cells were maintained in flasks coated with collagen. Cells were maintained at $37^{\circ} \mathrm{C}$ in a humidified atmosphere containing $5 \% \mathrm{CO}_{2}$. All cells tested negative for mycoplasma infection using MycoAlert Detection kit (Lonza) according to manufacturer's protocol. A4573-R and TC71-R cell lines were developed by adding YK to the growth media at the concentration equivalent to the $\mathrm{IC}_{50}$ of sensitive cells. As resistant cells regained normal growth rates in the presence of $\mathrm{YK}$, a new $\mathrm{IC}_{50}$ value was determined and the concentration of $\mathrm{YK}$ in the growth media was increased to $90 \%$ of the $\mathrm{IC}_{50}$ value. This was repeated until either a desirable degree of resistance was achieved (A4573-R) or the cells could no longer tolerate higher concentrations (TC71-R). All cell lines were passaged upon reaching $75-85 \%$ confluence at a ratio of $1: 10$. Resistant cell lines were passaged upon reaching $80-90 \%$ confluence at a ratio of 1:3 while increasing $\mathrm{YK}$ concentration and at a ratio of 1:5 once a stable YK concentration was reached.

Cell viability assays. Cell viability assays were performed using WST-1 (MilliporeSigma) according to the manufacturer's protocol. For 24-h compound treatment, cells were plated in a 96-well plate at a density of 10,000 cells/well for sensitive cells and 15,000 cells/well for resistant cells. Twenty-four-hour treatment was performed for YK, doxorubicin, and etoposide in $100 \mu \mathrm{l}$ volumes. For 48 -h treatment, cells were plated in a 96-well plate at a density of 5,000 cells/well for sensitive cells and 10,000 cells/well for resistant cells. Forty-eight-hour treatment was performed for vincristine, and imatinib in $200 \mu \mathrm{l}$ volumes.

RNA isolation and RT- $q P C R$. RNA was isolated using RNeasy Mini kit (Qiagen) according to the manufacturer's protocol. Reverse transcription (RT) was performed to produce cDNA using SuperScript VILO cDNA Synthesis Kit (Invitrogen; Thermo Fisher Scientific, Inc.) according to the manufacturer's protocol. Quantitative PCR (qPCR) was performed on a Roche LightCycler 480 II (Basel) using KiCqStart SYBR Green qPCR ReadyMix (MilliporeSigma) per the manufacturer's protocol. Primer pairs used for qPCR are listed in Table SI. A PCR profile of $95^{\circ} \mathrm{C}$ for $10 \mathrm{~min}(1$ cycle $), 95^{\circ} \mathrm{C}$ for $15 \mathrm{sec}$ followed by $59^{\circ} \mathrm{C}$ for $30 \mathrm{sec}$ followed by $72^{\circ} \mathrm{C}$ for $40 \mathrm{sec}$ ( $40 \mathrm{cycles}$ ), $95^{\circ} \mathrm{C}$ for $5 \mathrm{sec}$ followed by $65^{\circ} \mathrm{C}$ for $1 \mathrm{~min}$ (1 cycle) was used. Data were analyzed for expression relative to $18 \mathrm{~S}$ rRNA using the $2^{-\Delta \Delta \mathrm{Cq}}$ method (28).

RNA sequencing and analysis. RNA was isolated using a RNeasy Mini kit (Qiagen) according to manufacturer protocol. Sequencing libraries were generated from total mRNA using a TruSeq RNA Library Prep kit (Illumina) and sequenced on an Illumina HiSeq 2500 (Illumina). Reads were trimmed and adapters were removed using trimmomatic (29). Trimmed reads were aligned using STAR to human genome version GRCh38 with GENCODE annotation v27 (30). Unstranded gene-wise counts were assessed using htseq-count with the union parameter. Differential expression was assessed using the R package edgeR (31).

Orthotopic mouse xenograft model. A4573 xenograft tumors treated with YK were obtained from a previously published mouse study (21). Tumor samples were flash frozen in liquid nitrogen and stored at $-80^{\circ} \mathrm{C}$ until time of protein extraction for western blot analysis. 
Cell lysis, SDS-PAGE, and western blotting. Cells in culture were lysed using phospholysis buffer (50 mM HEPES pH 7.9, $100 \mathrm{mM}$ sodium chloride, $4.0 \mathrm{mM}$ sodium pyrophosphate, $10 \mathrm{mM}$ EDTA, $10 \mathrm{mM}$ sodium fluoride, and $1 \%$ Triton X-100 v:v) containing $2.0 \mathrm{mM}$ sodium vanadate, $1.0 \mathrm{mM} \mathrm{PMSF}, 4.0 \mu \mathrm{g} / \mathrm{ml}$ aprotinin, $4.0 \mu \mathrm{g} / \mathrm{ml}$ leupeptin, and $1.0 \mu \mathrm{g} / \mathrm{ml}$ calyculin A. For tumor tissue, between $60-80 \mathrm{mg}$ of frozen tissue was homogenized with a mortar and pestle with liquid nitrogen base and lysed using phospholysis buffer. Protein concentration was determined by bicinchoninic (BCA) assay using Pierce BCA Protein assay reagents (Thermo Fisher Scientific, Inc.) following the manufacturer's protocol. Samples were denatured after adding the appropriate volume of $5 \mathrm{X}$ Laemmli buffer. Concentrations and volume of samples were equalized using 1X Laemmli buffer prior to electrophoresis. Gels (12\% polyacrylamide) were run at $150 \mathrm{~V}$ for $1.5-2 \mathrm{~h}$. Protein transfer to Immobilon-P PVDF membrane (MilliporeSigma) was performed at $4^{\circ} \mathrm{C}$ either overnight at $330 \mathrm{mAmp}$ or for $3 \mathrm{~h}$ at 1,000 mAmp. Membranes were blocked in 5\% non-fat dry milk in 1X TTBS (20 mM Tris-HCl, pH 7.5, $150 \mathrm{mM} \mathrm{NaCl}$, $0.5 \%$ Tween-20 v:v) for one hour at room temperature. Three, five-minute washes were performed with 1X TTBS. Primary antibody was diluted 1:1,000 in 5\% BSA (MilliporeSigma) in $1 \mathrm{X}$ TTBS and incubated overnight at $4^{\circ} \mathrm{C}$. Primary antibodies used were anti-CD99 (ab75858, rabbit monoclonal, Abcam) and anti-FLI1 (MBS301248, rabbit polyclonal, MyBioSource). Three, five-minute washes were performed with $1 X$ TTBS. Secondary rabbit antibody (NA934-1ML, GE Healthcare) was diluted 1:5,000 in 5\% non-fat dry milk in 1X TTBS and incubated for one hour at room temperature. HRP-conjugated actin antibody (sc-1615, Santa Cruz Biotechnology, Inc.) was diluted $1: 5,000$ in $5 \%$ non-fat dry milk in $1 \mathrm{X}$ TTBS was incubated for $2 \mathrm{~h}$ at room temperature following blocking. Blots were developed using Immobilon Western Chemiluminescent HRP Substrate (MilliporeSigma) according to the manufacturer's protocol. A Fujifilm LAS-3000 system was used to detect chemiluminescence and image blots. To reblot membranes if needed, antibodies were stripped using Restore Western Blot Stripping Buffer (Thermo Fisher Scientific, Inc.) and the blotting process was repeated no more than one time. Protein quantification was performed using open source ImageJ software.

Statistical analyses. All statistical analyses were performed using GraphPad Prism software, version 8.0.2 (GraphPad Software, Inc.). All data are presented as the mean \pm standard error of the mean. $\mathrm{IC}_{50}$ values were determined by non-linear regression. The statistical difference between $\mathrm{IC}_{50}$ values was determined by Student's t-test or ordinary one-way analysis of variance with a Tukey's multiple comparisons test. The statistical difference between gene expression levels was determined by one-way analysis of variance with a Tukey's multiple comparisons test.

\section{Results}

A4573 cells developed robust resistance to $Y K$. In order to investigate potential molecular mechanisms that may lead to YK resistance in ES, we attempted to develop two YK resistant ES cell lines (A4573-R and TC71-R). YK was added to the growth media at the concentration equivalent to the $\mathrm{IC}_{50}$ of
A

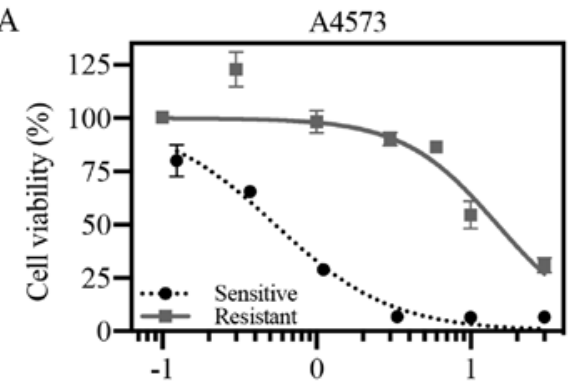

$\log [\mathrm{YK}$ concentration $(\mu \mathrm{M})]$

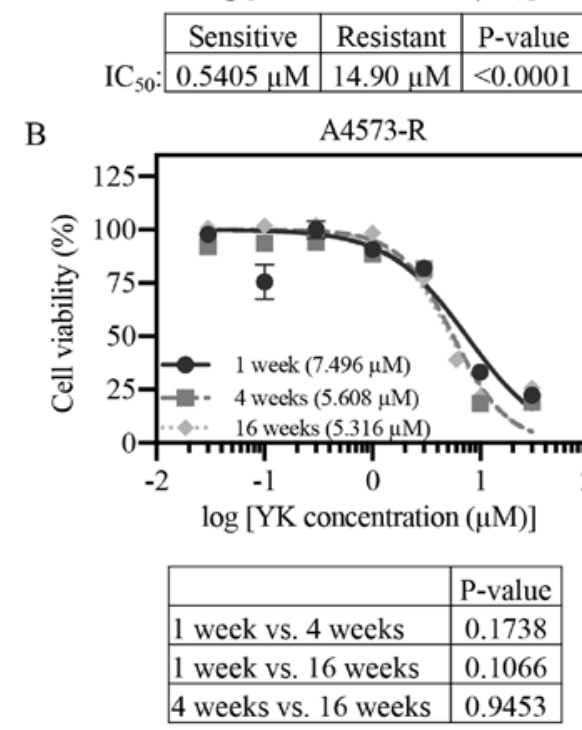

Figure 1. Development of a YK resistant A4573-R (A4573-R) cell line. (A) A4573 cells developed a significant resistance to YK, exhibiting a near 30-fold increase in $\mathrm{IC}_{50}$. (B) A4573-R cells maintained resistance to $\mathrm{YK}$ in the absence of the compound in media for 16 weeks. $\mathrm{IC}_{50}$ values for 1 week, 4 weeks and 16 weeks are presented. YK, YK-4-279; R, resistant.

A4573 $(0.54 \mu \mathrm{M})$ and TC71 $(0.88 \mu \mathrm{M})$ cell lines. As resistant cells regained normal growth rates in the presence of $\mathrm{YK}$, a new $\mathrm{IC}_{50}$ value was determined and the concentration of $\mathrm{YK}$ in the growth media was increased to $90 \%$ of the new $\mathrm{IC}_{50}$ value. Both A4573-R and TC71-R were able to tolerate increasing doses of YK until a desirable level of resistance was achieved or higher YK concentrations could no longer be tolerated (Fig. S1). A4573-R was utilized as the primary cell line for all subsequent experiments. After 550 days in culture with YK, A4573-R demonstrated significant resistance to $\mathrm{YK}$ as compared to sensitive $\mathrm{A} 4573$ cells (Fig. $1 \mathrm{~A}$, sensitive $\mathrm{IC}_{50}, 0.54 \mu \mathrm{M}$; resistant $\left.\mathrm{IC}_{50}, 14.9 \mu \mathrm{M} ; \mathrm{P}<0.0001\right)$. TC71 cells were less efficient in resistance development. Even though they showed an initial 7-fold increase in $\mathrm{IC}_{50}$ values (Fig. S1), they did not maintain that level of resistance. By day 360 the $\mathrm{IC}_{50}$ for TC71-R cells was $1.9 \mu \mathrm{M}$ compared to $0.6 \mu \mathrm{M}$ of sensitive cells (Fig. S2). In order to test if the resistance was maintained in the absence of $\mathrm{YK}$ in A4573-R cells, viability assays were performed at various points following YK removal from the media. We observed a decrease in $\mathrm{IC}_{50}$ values over 16 weeks in drug-free media. However, A4573-R cells still maintained significant resistance to YK compared to sensitive cells (Fig. 1B).

Resistance to $Y K$ is specific. A common mechanism for cancer cells to gain drug resistance is increased expression and/or 


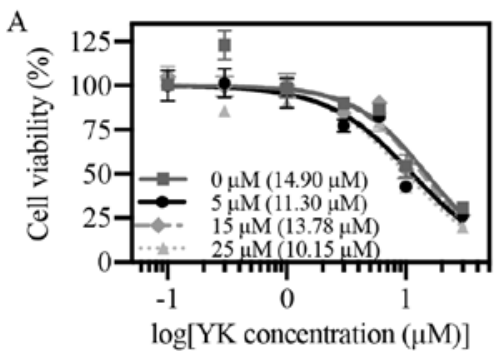

$\log [\mathrm{YK}$ concentration $(\mu \mathrm{M})]$

\begin{tabular}{|l|c|}
\hline & P-value \\
\hline $0 \mu \mathrm{M}$ vs. $5 \mu \mathrm{M}$ & 0.2924 \\
\hline $0 \mu \mathrm{M}$ vs. $15 \mu \mathrm{M}$ & 0.9492 \\
\hline $0 \mu \mathrm{M}$ vs. $25 \mu \mathrm{M}$ & 0.093 \\
\hline
\end{tabular}

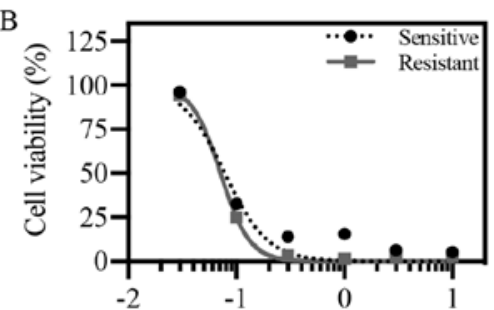

$\log [$ Doxorubicin concentration $(\mu \mathrm{M})]$

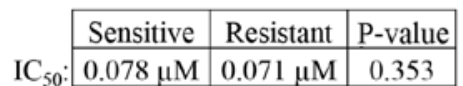

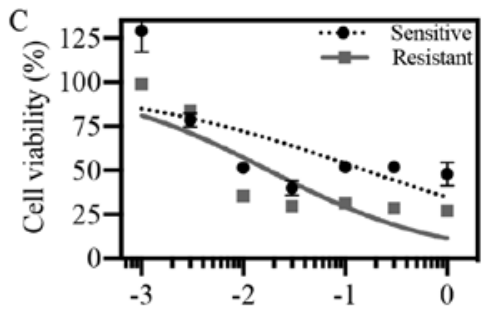

$\log [$ Vincristine concentration $(\mu \mathrm{M})]$

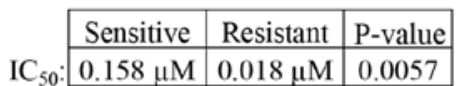

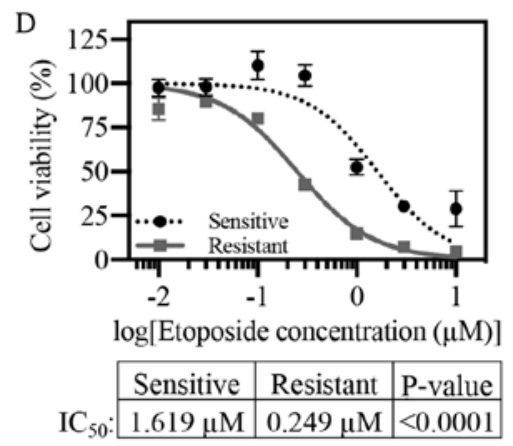

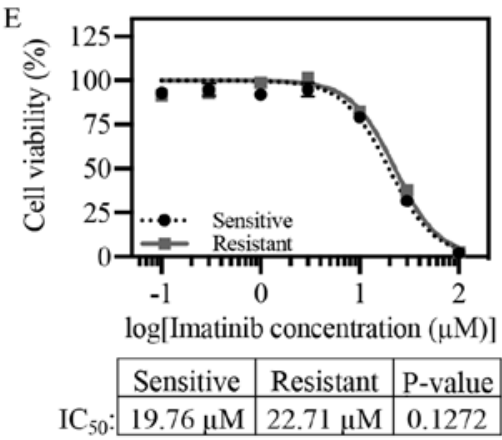

Figure 2. A4573-R resistance to YK is specific. (A) Treatment with increasing concentrations of the p-glycoprotein pump inhibitor, verapamil, does not affect A4573-R's sensitivity to YK. IC $\mathrm{I}_{50}$ values for each condition are presented. Viability curves comparing sensitive and resistant cell lines with (B) doxorubicin, (C) vincristine, (D) etoposide and (E) imatinib are provided. A4573-R cells did not exhibit cross-resistance to any of these compounds. R, resistant; YK, YK-4-279.

activity of efflux pumps, such as P-glycoprotein, which can provide resistance to multiple chemotherapeutic agents. In order to test if this mechanism was involved in YK resistance, viability assays were first performed using the efflux pump P-glycoprotein inhibitor verapamil in combination with YK. A4573-R did not display heightened sensitivity to YK with increasing verapamil treatment (Fig. 2A). This finding suggests that A4573-R resistance to $\mathrm{YK}$ is unlikely a result of increased P-glycoprotein efflux pump expression and/or activity. However, there are a number of mechanisms through which cells may develop multidrug resistance. Our data does not rule out these possibilities which need to be investigated further. Further, A4573-R cells did not exhibit cross resistance to three of the ES standard of care drugs: Doxorubicin, vincristine, and etoposide (Figs. 2B-D and S2B-D for TC71). There was even increased sensitivity to etoposide and vincristine in the A4573-R cell line. In a similar study characterizing ES resistance mechanisms to YK, Lamhamedi-Cherradi et al reported that YK resistant cell lines were cross resistant to the tyrosine kinase inhibitor imatinib (32). In our study, A4573-R cells did not display cross resistance to imatinib (Fig. 2E).

EWS-FLII expression is unchanged and CD99 expression is increased in resistant cells. To address the possibility that resistance is due to differential expression of EWS-FLI1, the target of YK, EWS-FLI1 expression was quantified at both the RNA and protein levels. Expression of CD99 was also assessed, as this protein is important in ES biology. RNA expression of these two genes was evaluated longitudinally: At days 236, 437 and 584 for A4573-R (Fig. 3A),

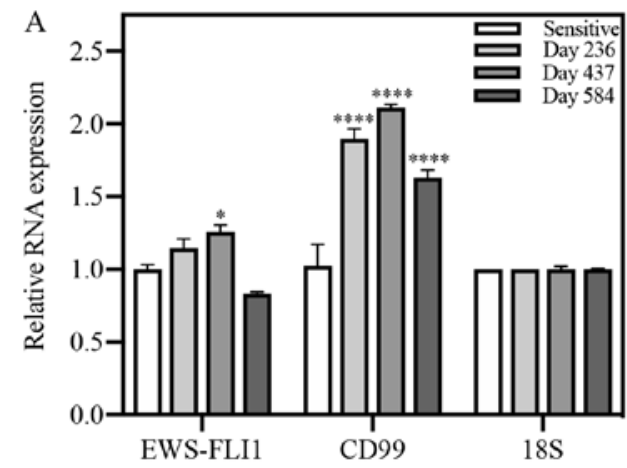

B

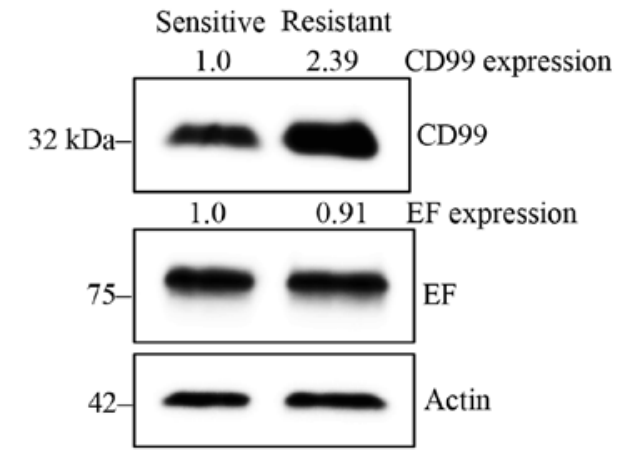

Figure 3. CD99 expression is increased in A4573-R cells. (A) Quantitative PCR analysis was performed for EF, CD99 and 18S ribosomal RNA in A4573 sensitive cells and resistant cells at multiple timepoints. CD99 RNA expression is significantly elevated compared with sensitive cells at all timepoints. ${ }^{*} \mathrm{P}<0.05$ and ${ }^{* * * *} \mathrm{P}<0.0001$ vs. sensitive. (B) Western blot anlaysis of CD99 and $\mathrm{EF}$ in A4573 sensitive and resistant cells. Expression levels were calculated using densitometry analysis relative to sensitive cell lines. CD99 expression is elevated in A4573-R cells. R, resistant; EF, EWS-FLI1. 
A

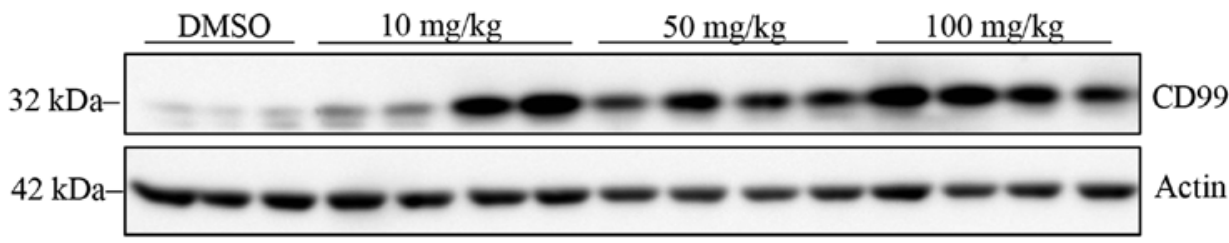

B

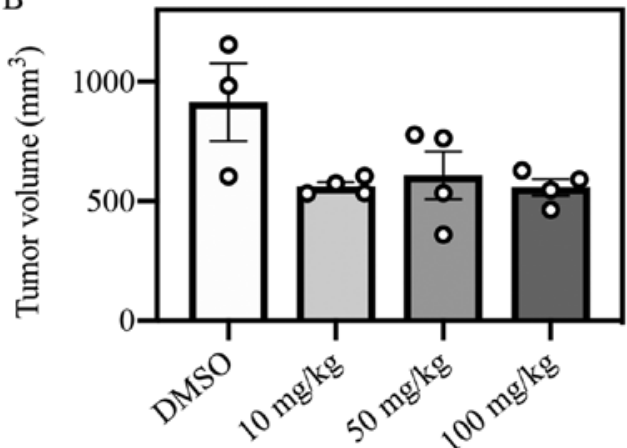

C

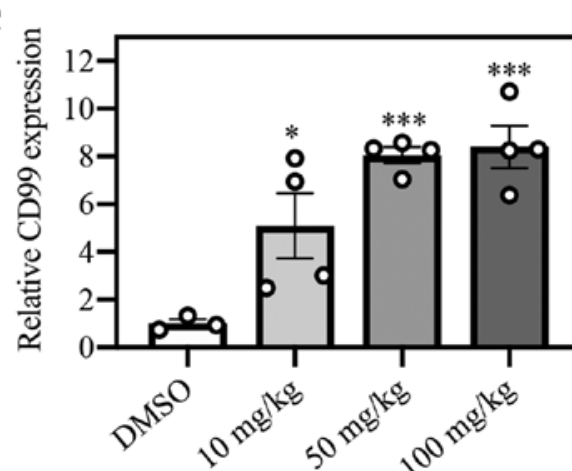

Figure 4. YK treatment of A4573 xenografts results in an elevated expression of CD99 in vivo. (A) Western blot analysis for CD99 expression in tumors collected after 5 days of YK treatment at concentrations of 10,50 and $100 \mathrm{mg} / \mathrm{kg}$, or DMSO control treatment. (B) Tumor volume at endpoint. No significant difference was identified between the groups. (C) Densitometry quantification of western blotting relative to DMSO control tumor expression. Circles represent individual samples. CD99 expression was significantly elevated in all treatment groups. ${ }^{*} \mathrm{P}<0.05$ and ${ }^{* * * *} \mathrm{P}<0.001$ vs. DMSO. YK, YK-4-279.

A
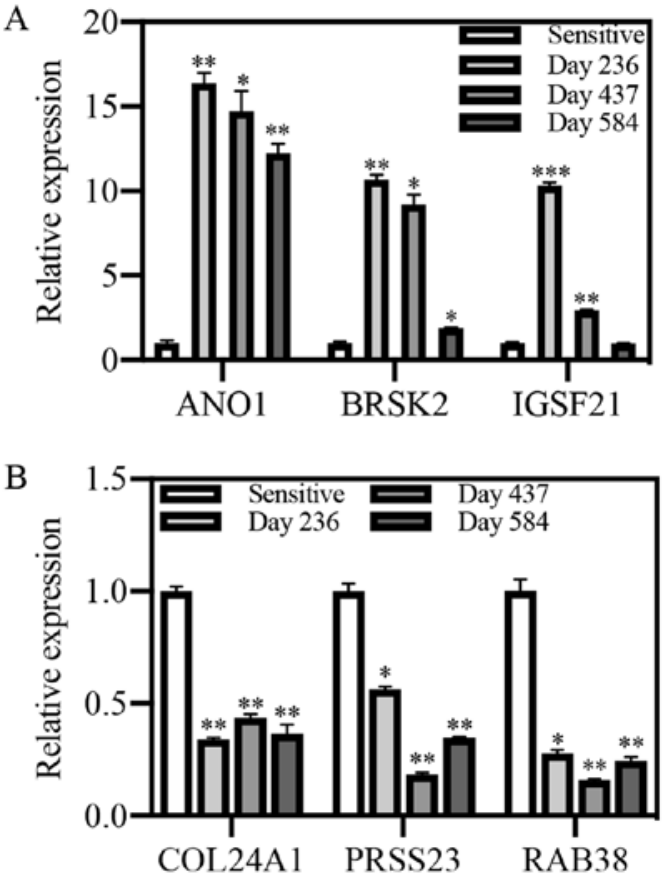

Figure 5. Quantitative PCR for upregulated genes identified through RNA sequencing in A4573 cells. Relative expression was evaluated at multiple time points in resistant cells compared with sensitive cells. (A) ANO1, BRSK2, and IGSF21 were upregulated. (B) COL24A1, PRSS23 and RAB38 were downregulated. ${ }^{*} \mathrm{P}<0.05,{ }^{* *} \mathrm{P}<0.01$ and ${ }^{* * *} \mathrm{P}<0.001$ vs. sensitive. ANO1, anoctamin 1; BRSK2, BR serine/threonine kinase 2; IGSF21, immunoglobulin superfamily member 21; COL24A1, collagen type XXIV alpha 1 chain; PRSS23, serine protease 23; RAB38, Ras-related protein Rab-38.

and at days 181, 342 and 489 for TC71-R (Fig. S3A). CD99 RNA expression was consistently increased significantly as compared to sensitive cells at all time points evaluated. EWS-FLI1 RNA expression was only significantly increased at day 437 for A4573 and day 342 for TC71, and returned to levels not significantly different from sensitive cells at the following time point for both cell lines. EWS-FLI1 and CD99 protein expression was also evaluated in both cell lines. Relative to sensitive cells, CD99 protein expression was increased nearly 2.4-fold in A4573-R cells (Fig. 3B). Similarly, TC71-R exhibited a 1.75-fold increase in CD99 protein expression (Fig. S3B). EWS-FLI1 protein expression does not appear to be affected by acquisition of resistance.

CD99 expression is elevated within five days of YK treatment in vivo. Mice with sensitive A4573 xenograft tumors were treated with YK by IP injection daily over the course of five days. DMSO was used as vehicle control, and three escalating dose groups of YK were observed: 10,50 and $100 \mathrm{mg} / \mathrm{kg}$. All YK-treated groups exhibited elevated levels of CD99 protein expression vs. the DMSO control group (Fig. 4A and C). Tumor volumes between the DMSO control and YK-treated groups did not significantly differ after five days of treatment (Fig. 4B). These data suggest that elevation of CD99 expression is an early effect of YK treatment and subsequent development of resistance. We tried to inhibit CD99 expression by siRNA and perform drug sensitivity studies in resistant cells but we could not achieve good reduction in protein levels. Since inhibition of CD99 results in death of ES cells, this finding was not surprising.

RNA sequencing revealed candidate genes involved in resistance mechanisms and expression was validated by $R T$ - $q P C R$. RNA sequencing of A4573 and TC71 sensitive and resistant cells was performed on day 236 and 181 of culture respectively to identify differentially expressed genes. For each cell line, data sets were filtered for genes with at least a 2-fold change in expression. The A4573 and TC71 filtered data sets were compared to determine the genes with expression changes that were shared between the two cell lines. From this pool of 152 genes (Table SII), a second filter of 4-fold change 
in expression was applied in order to narrow down potential candidates. Expression of these candidate genes was evaluated in resistant cells. Three upregulated and three downregulated genes were validated in triplicate by RT-qPCR in both A4573 (Fig. 5) and TC71 (Fig. S4).

The three top upregulated genes were ANO1, BRSK2, and IGSF21, and the three top downregulated genes were COL24A1, PRSS23, and RAB38. Expression of all three candidate upregulated genes, ANO1, BRSK2, and IGSF21, showed significant upregulation by $\mathrm{qPCR}$ at the time of RNA sequencing in A4573-R cells (Fig. 5A). High ANO1 expression was maintained in both cell lines over time. Expression of all three candidate down-regulated genes, COL24A1, PRSS23, and RAB38, displayed significant down-regulation at all timepoints in A4573-R cells as compared to sensitive cell expression (Fig. 5B). Further mechanistic investigation of these gene expression changes is needed.

\section{Discussion}

This study created cell lines that are resistant to YK for purposes of planning future combinatorial studies to avoid single agent resistance. In this body of work, we attempted to establish resistance to the EWS-FLI1 inhibitor (S)-YK in two of the commonly used ES cell lines A4573 and TC71. These two cell lines were chosen because they each harbor a different version of the EWS-FLI1 fusion; TC71 has a type 1 fusion and A4573 has a type 3 fusion. The cell line A4573 developed sustained, and specific resistance to YK over time to levels that was consistent with published resistance models (33). However, the resistance in cell line TC71 was not as stable and over a 1-year period of time did not show a significant resistant phenotype. Future work will also include combinatorial testing with compounds known to be synergistic with YK (21).

No cross-resistance was observed to any of the current standard-of-care drugs that were tested. Also, resistant cells did not become more sensitive to YK when treated in combination with the p-glycoprotein inhibitor verapamil, indicating that the efflux pump p-glycoprotein, which is often associated with multidrug resistance mechanisms, is not the main driver of resistance in A4573-R cells. Cancer cells can acquire multidrug resistance through multiple mechanisms. Measurement of YK concentration within A4573-R cells by mass spectrometry and comparing it to sensitive A4573 cells may provide evidence for augmented drug metabolism or accelerated efflux of the drug by an alternate pathway. A4573-R cells exhibited an increased sensitivity to the standard-of-care drugs etoposide and vincristine. We did not observe the same phenotype in TC71-R cells. Since YK and vincristine show synergy (21), A4573-R cells that have stronger and sustainable YK resistance may also have acquired better sensitivity to etoposide and vincristine compared to the TC71-R cells that had a weaker YK resistance. Alternatively, the observed difference may be an artifact of selection during development of resistance and maintenance.

An avenue that was not explored through this study was the possibility that resistance emerged due to a mutation in EWS-FLI1. Sequencing of both sensitive and resistant cells could be performed to evaluate EWS-FLI1 mutation status. Any mutation identified would need to be validated by re-development of resistant lines, as the current cell lines have been in culture with YK for over a year and it would be impossible to decipher if resistance arose due to the mutation. In the developed resistant lines, YK viability assays could be performed in conjunction with DNA collection to determine if the acquisition of resistance coincides with the mutation presence. Any mutations in EWS-FLI1 that arise simultaneously with acquired resistance may be associated with binding site of YK, and could help shed light on the location of YK binding to the EWS-FLI1 fusion protein.

There is another publication characterizing ES resistance to YK. While resistance to YK was established, Lamhamedi-Cherradi et al found that resistant cells became cross-resistant to the tyrosine kinase inhibitor imatinib (32). Despite creating a resistant cell line with a similar level of resistance to YK ( 28 -fold increase), the YK resistant cells developed in this body of work did not demonstrate cross resistance to imatinib. One reason this difference might have occurred is the method by which the cells were made resistant. In the previous study, sensitive cells were exposed to a high $3 \mu \mathrm{M}$ concentration of $\mathrm{YK}$ and subsequently maintained at that concentration for an unspecified amount of time. In this study, the concentration of YK in the growth media was gradually increased over time as tolerated by the cells, and maintained in culture for $>1$ year. This distinct difference in development of resistant cell lines may be inadvertently highlighting the differences between cells that are intrinsically resistant vs. those that acquire resistance. In the context of a tumor, cells with intrinsic resistance exist, and more cells will acquire resistance as treatment is sustained (34). By exposing ES cells to especially high concentrations of YK, Lamhamedi-Cherradi et al (32) created clonal populations of cells, which presumably harbor intrinsic resistance mechanisms to YK. One of those mechanisms potentially also contributed to cross-resistance of imatinib. In this study, gradually increasing the concentration of YK present created resistant populations of cells by allowing cells to acquire resistance to the drug. While there may be a proportion of cells in this resistant population that possess an intrinsic resistance to imatinib, the data presented here do not demonstrate that possession of this capability is necessary for resistance to YK. However, resistance to imatinib should be an important consideration when determining revised treatment strategies in eventual cases of TK216 resistance in patients.

Even though the $\mathrm{IC}_{50}$ values were gradually increasing, resistant A4573 cells were able to maintain the phenotype in the absence of YK for up to 16 weeks. This signals that the resistant population acquired changes that facilitated resistance to but were independent of YK treatment. In other words, the mechanisms driving YK resistance became fixed cellular processes after an extended period of exposure to YK. These data and the current structure of treatment highlight the necessity of understanding the driving mechanisms of resistance. An important caveat to note is that resistant cells were in culture with YK for over one year at the time the washout experiment began. This is a long period of time for cells and may not accurately reflect changes that occur in humans with ES. Future experiments are required to determine if this phenomenon exists in cells exposed to YK for shorter periods of time. 
CD99 unexpectedly emerged as an upregulated protein in YK resistant cells. CD99 RNA and protein expression was elevated in both the A4573-R and TC71-R cell lines. A4573 xenograft tumors in vivo exhibited elevated CD99 expression after just five days of YK treatment with no differences in tumor volume. This five-day evaluation of xenograft tumors was done as an early phase pharmacodynamic marker in a previous study (21). We do not believe that these xenograft tumors are resistant to YK yet. We have performed several mouse xenograft studies with ES cell lines (including A4573) and YK $(18,20)$. In control groups, the tumor volume grew exponentially but in the YK treatment groups the tumor volume stayed close to unchanged. Through histopathological analysis we observed increased apoptosis, reduced mitosis, and reduced expression of EWS-FLI1 target genes in these tumors (20). Therefore, there was a clear and significant drug effect on tumor growth. Lack of tumor shrinkage was primarily due to the relatively poor pharmacokinetic profile of YK. When we performed similar studies in rats with ES cell line xenografts, we observed tumor shrinkage because we were able to deliver the drug through a continuous IV infusion. In mouse studies, IP or IV bolus injections do not deliver enough drug to maintain serum levels that would cause tumor shrinkage. The patients in the current clinical trial are receiving the drug with continuous IV injection for 14-21 days.

There is a correlation between EWS-FLI1 expression and CD99 in ES cell lines. The CD99 promoter contains a consensus ets binding site, which shows EWS-FLI1 binding by ChIP assay (35). There is evidence that EWS-FLI1 may induce CD99 expression (36-38). Therefore, one might expect to see reduced CD99 expression following inhibition of EWS-FLI1 activity by YK. However, the observed increase in CD99 expression in A4573-R cells suggest a compensation mechanism, which further highlights the biological importance of CD99 for ES cell survival.

Increased CD99 expression has been reported previously in a resistant population of cells, which poses an intriguing question surrounding the role of CD99 in resistance mechanisms. Aldegaither and colleagues developed a squamous cell carcinoma line that was resistant to antibody-dependent cell-mediated cytotoxicity (ADCC) (39). Under ADCC conditions, targeting antibodies direct binding of effector natural killer cells to cancer cells to create an immune synapse. Upon effector cell binding to the cancer cells through the target antibody, the effector cells release perforins and granzymes, which cause apoptosis to occur in the cancer cell. In their model system, CD99 expression is increased 16-fold in the ADCC resistant cells. Their study did not explore the role of CD99 in maintenance of the resistant phenotype. This study did not explore the mechanisms through which YK treatment induced high CD99 expression. It would be intriguing to assess if CD99 is playing a role in YK resistance specifically or has a role in more general resistance mechanisms. Cell lines could be established that are resistant to other drugs and CD99 expression could be evaluated. If CD99 expression is elevated, this would suggest that a role for CD99 exists in a broader context of resistance.

RNA sequencing of resistant and sensitive cells revealed six top candidate genes that may be involved in YK resistance mechanisms, and their expression was validated in both A4573 and TC71 resistant cell lines by qPCR. The top upregulated genes are the calcium activated chloride channel ANO1, the serine/threonine kinase BRSK2, and the immunoglobulin family member IGSF21. The top downregulated genes are the collagen COL24A1, the serine protease PRSS23, and the Ras-related GTPase RAB38. The threshold for candidate genes to validate by qPCR was set high at a 4-fold change cut-off mark. These six genes consistently exhibited expected expression levels in resistant cells at all points in time evaluated for A4573, with the one exception being IGSF21 at the latest time point. There were some inconsistencies in TC71-R expression of BRSK2 and PRSS23. For BRSK2, gene expression did not match what was observed by RNA sequencing, but expression increased as time progressed. For PRSS23, expression was not downregulated at any of the timepoints beyond RNA sequencing at day 181. This may be due to a change in culture conditions following RNA collection for RNA sequencing-cells were not thriving on plastic and were transferred to culture in collagen-coated flasks for all points forward. It may be possible that of the 149 genes returned after the initial cut off of 2-fold change would have been validated by $\mathrm{qPCR}$ and are important in resistance mechanisms. A larger qPCR screen would be required to make this determination.

Of the validated candidate genes, one that could be interesting to investigate further would be ANO1. ANO1 is utilized as a diagnostic marker in gastrointestinal stromal tumors, but its functional role is still under investigation (40-42). Interestingly, ANO1 has been functionally associated with c-kit, which was one of the genes identified by Lamhamedi-Cherradi et al (32) to be upregulated in their YK resistant cells (43). Given that CD99 is a critical player in resistance mechanisms and has been associated with ion channels previously (44), it would be interesting to see if ANO1 is associated with CD99-dependent YK resistance in ES cells. Knockdown of ANO1 by siRNA in resistant cells and subsequent assessment of sensitivity to YK would need to be performed to determine if this protein is also important in YK resistance mechanisms.

We developed a model of YK resistance in A4573 ES cells in culture. The cell line maintained a stable resistance that was not consistent in both cell lines, so this opens future opportunities for mechanistic investigation. Several genes were identified that may have a role in resistance mechanisms. Future studies are needed to determine their significance, particularly ANO1. Overall, this study contributes new information regarding the biology of ES and may lead to a better understanding of CD99.

\section{Acknowledgements}

Not applicable.

Funding

No funding was received.

\section{Availability of data and materials}

The datasets used and/or analyzed during the current study are available from the corresponding author on reasonable request. 


\section{Authors' contributions}

AÜ and EC conceived and designed the present study. EC, SH, DA, GG, JP, MKB, SS and SHH performed the experiments. EC, SH, DA, GG, SS, HÇ, JT and AÜ analyzed the data. SS and HÇ contributed reagents/technical expertise/materials/intellectual discussion/analysis tools. EC and AÜ wrote the manuscript. All authors reviewed, edited and approved the manuscript.

\section{Ethics approval and consent to participate}

Not applicable.

\section{Patient consent for publication}

Not applicable.

\section{Competing interests}

United States Patent and Trademark Office awarded a patent for YK-4-279 to Georgetown University, inventors include AÜ and JAT (Patent no. 8,232,310). The other authors declare that they have no competing interests.

\section{References}

1. Balamuth NJ and Womer RB: Ewing's sarcoma. Lancet Oncol 11: 184-192, 2010.

2. Aurias A, Rimbaut C, Buffe D, Dubousset J and Mazabraud A: Chromosomal translocations in Ewing's sarcoma. N Engl J Med 309: 496-498, 1983.

3. Delattre O, Zucman J, Plougastel B, Desmaze C, Melot T, Peter M Kovar H, Joubert I, de Jong P, Rouleau G, et al: Gene fusion with an ETS DNA-binding domain caused by chromosome translocation in human tumours. Nature 359: 162-165, 1992.

4. Delattre O, Zucman J, Melot T, Garau XS, Zucker JM, Lenoir GM, Ambros PF, Sheer D, Turc-Carel C, Triche TJ, et al: The Ewing family of tumors-a subgroup of small-round-cell tumors defined by specific chimeric transcripts. N Engl J Med 331: 294-299, 1994.

5. May WA, Gishizky ML, Lessnick SL, Lunsford LB, Lewis BC, Delattre O, Zucman J, Thomas G and Denny CT: Ewing sarcoma $11 ; 22$ translocation produces a chimeric transcription factor that requires the DNA-binding domain encoded by FLI1 for transformation. Proc Natl Acad Sci USA 90: 5752-5756, 1993.

6. Jaishankar S, Zhang J, Roussel MF and Baker SJ: Transforming activity of EWS/FLI is not strictly dependent upon DNA-binding activity. Oncogene 18: 5592-5597, 1999.

7. Chan D, Wilson TJ, Xu D, Cowdery HE, Sanij E, Hertzog PJ and Kola I: Transformation induced by Ewing's sarcoma associated EWS/FLI-1 is suppressed by KRAB/FLI-1. Br J Cancer 88: 137-145, 2003.

8. May WA, Lessnick SL, Braun BS, Klemsz M, Lewis BC, Lunsford LB, Hromas R and Denny CT: The Ewing's sarcoma EWS/FLI-1 fusion gene encodes a more potent transcriptional activator and is a more powerful transforming gene than FLI-1. Mol Cell Biol 13: 7393-7398, 1993.

9. Ramakrishnan R, Fujimura Y, Zou JP, Liu F, Lee L, Rao VN and Reddy ES: Role of protein-protein interactions in the antiapoptotic function of EWS-Fli-1. Oncogene 23: 7087-7094, 2004.

10. Ohno T, Rao VN and Reddy ES: EWS/Fli-1 chimeric protein is a transcriptional activator. Cancer Res 53: 5859-5863, 1993.

11. Toretsky JA, Erkizan V, Levenson A, Abaan OD, Parvin JD, Cripe TP, Rice AM, Lee SB and Uren A: Oncoprotein EWS-FLI1 activity is enhanced by RNA helicase A. Cancer Res 66: 5574-5581, 2006.

12. Erkizan HV, Schneider JA, Sajwan K, Graham GT, Griffin B, Chasovskikh S, Youbi SE, Kallarakal A, Chruszcz M, Padmanabhan R, et al: RNA helicase A activity is inhibited by oncogenic transcription factor EWS-FLI1. Nucleic Acids Res 43: 1069-1080, 2015.
13. Yang L, Chansky HA and Hickstein DD: EWS.Fli-1 fusion protein interacts with hyperphosphorylated RNA polymerase II and interferes with serine-arginine protein-mediated RNA splicing. J Biol Chem 275: 37612-37618, 2000.

14. Knoop LL and Baker SJ: The splicing factor U1C represses EWS/FLI-mediated transactivation. J Biol Chem 275: 24865-24871, 2000.

15. Knoop LL and Baker SJ: EWS/FLI alters 5'-splice site selection. J Biol Chem 276: 22317-22322, 2001.

16. Selvanathan SP, Graham GT, Erkizan HV, Dirksen U, Natarajan TG, Dakic A, Yu S, Liu X, Paulsen MT, Ljungman ME, et al: Oncogenic fusion protein EWS-FLI1 is a network hub that regulates alternative splicing. Proc Natl Acad Sci USA 112: E1307-E1316, 2015.

17. Sanchez G, Bittencourt D, Laud K, Barbier J, Delattre O, Auboeuf D and Dutertre M: Alteration of cyclin D1 transcript elongation by a mutated transcription factor up-regulates the oncogenic D1b splice isoform in cancer. Proc Natl Acad Sci USA 105: 6004-6009, 2008.

18. Erkizan HV, Kong Y, Merchant M, Schlottmann S, Barber-Rotenberg JS, Yuan L, Abaan OD, Chou TH, Dakshanamurthy S, Brown ML, et al: A small molecule blocking oncogenic protein EWS-FLI1 interaction with RNA helicase A inhibits growth of Ewing's sarcoma. Nat Med 15: 750-756, 2009.

19. Barber-Rotenberg JS, Selvanathan SP, Kong Y, Erkizan HV, Snyder TM, Hong SP, Kobs CL, South NL, Summer S, Monroe PJ, et al: Single enantiomer of YK-4-279 demonstrates specificity in targeting the oncogene EWS-FLI1. Oncotarget 3: 172-182, 2012.

20. Hong SH, Youbi SE, Hong SP, Kallakury B, Monroe P, Erkizan HV, Barber-Rotenberg JS, Houghton P, Üren A and Toretsky JA: Pharmacokinetic modeling optimizes inhibition of the 'undruggable' EWS-FLI1 transcription factor in Ewing sarcoma. Oncotarget 5: 338-350, 2014.

21. Zöllner SK, Selvanathan SP, Graham GT, Commins RMT, Hong SH, Moseley E, Parks S, Haladyna JN, Erkizan HV, Dirksen U, et al: Inhibition of the oncogenic fusion protein EWS-FLI1 causes $\mathrm{G}_{2}-\mathrm{M}$ cell cycle arrest and enhanced vincristine sensitivity in Ewing's sarcoma. Sci Signal 10: pii: eaam8429, 2017.

22. Rahim S, Beauchamp EM, Kong Y, Brown ML, Toretsky JA and Üren A: YK-4-279 inhibits ERG and ETV1 mediated prostate cancer cell invasion. PLoS One 6: e19343, 2011.

23. Rahim S, Minas T, Hong SH, Justvig S, Çelik H, Kont YS, Han J, Kallarakal AT, Kong Y, Rudek MA, et al: A small molecule inhibitor of ETV1, YK-4-279, prevents prostate cancer growth and metastasis in a mouse Xenograft model. PLoS One 9: e114260, 2014.

24. Winters B, Brown L, Coleman I, Nguyen H, Minas TZ, Kollath L, Vasioukhin V, Nelson P, Corey E, Üren A and Morrissey $\mathrm{C}$ : Inhibition of ERG activity in patient-derived prostate cancer Xenografts by YK-4-279. Anticancer Res 37: 3385-3396, 2017.

25. Sun W, Rojas Y, Wang H, Yu Y, Wang Y, Chen Z, Rajapakshe K, Xu X, Huang W, Agarwal S, et al: EWS-FLI1 and RNA helicase A interaction inhibitor YK-4-279 inhibits growth of neuroblastoma. Oncotarget 8: 94780-94792, 2017.

26. Kollareddy M, Sherrard A, Park JH, Szemes M, Gallacher K, Melegh Z, Oltean S, Michaelis M, Cinatl J Jr, Kaidi A and Malik K: The small molecule inhibitor YK-4-279 disrupts mitotic progression of neuroblastoma cells, overcomes drug resistance and synergizes with inhibitors of mitosis. Cancer Lett 403: 74-85, 2017.

27. TK216 in patients with relapsed or refractory Ewing sarcoma. NCT02657005. https://clinicaltrials.gov/ct2/show/ NCT02657005. Accessed January 15, 2016.

28. Livak KJ and Schmittgen TD: Analysis of relative gene expression data using real-time quantitative PCR and the 2(-Delta Delta $\mathrm{C}(\mathrm{T}))$ method. Methods 25: 402-408, 2001.

29. Bolger AM, Lohse M and Usadel B: Trimmomatic: A flexible trimmer for Illumina sequence data. Bioinformatics 30 : 2114-2120, 2014.

30. Dobin A, Davis CA, Schlesinger F, Drenkow J, Zaleski C, Jha S, Batut P, Chaisson M and Gingeras TR: STAR: Ultrafast universal RNA-seq aligner. Bioinformatics 29: 15-21, 2013.

31. Robinson MD, McCarthy DJ and Smyth GK: EdgeR: A Bioconductor package for differential expression analysis of digital gene expression data. Bioinformatics 26: 139-140, 2010. 
32. Lamhamedi-Cherradi SE, Menegaz BA, Ramamoorthy V, Aiyer RA, Maywald RL, Buford AS, Doolittle DK, Culotta KS, O'Dorisio JE and Ludwig JA: An oral formulation of YK-4-279: Preclinical efficacy and acquired resistance patterns in Ewing sarcoma. Mol Cancer Ther 14: 1591-1604, 2015.

33. McDermott M, Eustace AJ, Busschots S, Breen L, Crown J, Clynes M, O'Donovan N and Stordal B: In vitro development of chemotherapy and targeted therapy drug-resistant cancer cell lines: A practical guide with case studies. Front Oncol 4: 40, 2014.

34. Foo $\mathrm{J}$ and Michor F: Evolution of acquired resistance to anti-cancer therapy. J Theor Biol 355: 10-20, 2014.

35. Rocchi A, Manara MC, Sciandra M, Zambelli D, Nardi F, Nicoletti G, Garofalo C, Meschini S, Astolfi A, Colombo MP, et al: CD99 inhibits neural differentiation of human Ewing sarcoma cells and thereby contributes to oncogenesis. J Clin Invest 120 : 668-680, 2010

36. Lessnick SL, Dacwag CS and Golub TR: The Ewing's sarcoma oncoprotein EWS/FLI induces a p53-dependent growth arrest in primary human fibroblasts. Cancer Cell 1: 393-401, 2002

37. Hu-Lieskovan S, Zhang J, Wu L, Shimada H, Schofield DE and Triche TJ: EWS-FLI1 fusion protein up-regulates critical genes in neural crest development and is responsible for the observed phenotype of Ewing's family of tumors. Cancer Res 65: 4633-4644, 2005

38. Suvà ML, Riggi N, Stehle JC, Baumer K, Tercier S, Joseph JM, Suvà $\mathrm{D}$, Clément V, Provero $\mathrm{P}$, Cironi L, et al: Identification of cancer stem cells in Ewing's sarcoma. Cancer Res 69: 1776-1781, 2009.
39. Aldeghaither DS, Zahavi DJ, Murray JC, Fertig EJ, Graham GT, Zhang YW, O'Connell A, Ma J, Jablonski SA and Weiner LM: A mechanism of resistance to antibody-targeted immune attack. Cancer Immunol Res 7: 230-243, 2019.

40. Tuveson DA, Willis NA, Jacks T, Griffin JD, Singer S, Fletcher CD, Fletcher JA and Demetri GD: STI571 inactivation of the gastrointestinal stromal tumor c-KIT oncoprotein: Biological and clinical implications. Oncogene 20: 5054-5058, 2001.

41. Berglund E, Akcakaya P, Berglund D, Karlsson F, Vukojević V, Lee L, Bogdanović D, Lui WO, Larsson C, Zedenius J, et al: Functional role of the $\mathrm{Ca}^{2+}$-activated $\mathrm{Cl}^{-}$channel DOG1/TMEM16A in gastrointestinal stromal tumor cells. Exp Cell Res 326: 315-325, 2014.

42. Rizzo FM, Palmirotta R, Marzullo A, Resta N, Cives M, Tucci M and Silvestris F: Parallelism of DOG1 expression with recurrence risk in gastrointestinal stromal tumors bearing KIT or PDGFRA mutations. BMC Cancer 16: 87, 2016.

43. Simon S, Grabellus F, Ferrera L, Galietta L, Schwindenhammer B, Mühlenberg T, Taeger G, Eilers G, Treckmann J, Breitenbuecher F, et al: DOG1 regulates growth and IGFBP-5 in gastrointestinal stromal tumors. Cancer Res 73: 3661-3670, 2013.

44. Kreppel M, Aryee DN, Schaefer KL, Amann G, Kofler R, Poremba $\mathrm{C}$ and Kovar H: Suppression of KCMF1 by constitutive high CD99 expression is involved in the migratory ability of Ewing's sarcoma cells. Oncogene 25: 2795-2800, 2006. 